\title{
Phonon excitation and instabilities in biased graphene nanoconstrictions
}

\author{
Gunst, Tue; Lu, Jing Tao; Hedegård, Per; Brandbyge, Mads
}

Published in:

Physical Review B

Link to article, DOI:

10.1103/PhysRevB.88.161401

Publication date:

2013

Document Version

Publisher's PDF, also known as Version of record

Link back to DTU Orbit

Citation (APA):

Gunst, T., Lu, J. T., Hedegård, P., \& Brandbyge, M. (2013). Phonon excitation and instabilities in biased graphene nanoconstrictions. Physical Review B, 88(16), 161401. https://doi.org/10.1103/PhysRevB.88.161401

\section{General rights}

Copyright and moral rights for the publications made accessible in the public portal are retained by the authors and/or other copyright owners and it is a condition of accessing publications that users recognise and abide by the legal requirements associated with these rights.

- Users may download and print one copy of any publication from the public portal for the purpose of private study or research.

- You may not further distribute the material or use it for any profit-making activity or commercial gain

- You may freely distribute the URL identifying the publication in the public portal

If you believe that this document breaches copyright please contact us providing details, and we will remove access to the work immediately and investigate your claim. 


\title{
Phonon excitation and instabilities in biased graphene nanoconstrictions
}

\author{
Tue Gunst, ${ }^{1, *}$ Jing-Tao Lü, ${ }^{1,2, \dagger}$ Per Hedegård, ${ }^{2}$ and Mads Brandbyge ${ }^{1}$ \\ ${ }^{1}$ Department of Micro- and Nanotechnology (DTU Nanotech), Center for Nanostructured Graphene (CNG), \\ Technical University of Denmark, DK-2800 Kgs. Lyngby, Denmark \\ ${ }^{2}$ Niels Bohr Institute, Nano-Science Center, University of Copenhagen, Universitetsparken 5, 2100 Copenhagen $\emptyset$, Denmark
}

(Received 9 May 2013; published 11 October 2013)

\begin{abstract}
We investigate how a high current density perturbs the phonons in a biased graphene nanoconstriction coupled to semi-infinite electrodes. The coupling to electrode phonons, electrode electrons under bias, Joule heating, and current-induced forces is evaluated using first principles density functional theory and nonequilibrium Green's function calculations. We observe a strongly nonlinear heating of the phonons with bias and breakdown of the harmonic approximation when the Fermi level is tuned close to a resonance in the electronic structure of the constriction. This behavior is traced back to the presence of negatively damped phonons driven by the current. The effects may limit the stability and capacity of graphene nanoconstrictions to carry high currents.
\end{abstract}

DOI: 10.1103/PhysRevB.88.161401

PACS number(s): 63.22.Rc, 72.80.Vp, 72.10.Di, 46.32.+x

Graphene has emerged as a highly attractive material for future electronic devices. ${ }^{1,2}$ It can sustain current densities six orders of magnitude larger than copper and is foreseen to be a versatile material with numerous applications in nanoelectronics, spintronics, and nanoelectromechanics. ${ }^{3}$ In graphene nanoconstrictions (GNCs) the current is passed through a short ribbon ${ }^{4,5}$ at the narrowest point. Constrictions and nanoribbons provide semiconducting interconnects in graphene nanocircuitry, ${ }^{6,7}$ and is a central building block of graphene-based nanoelectronics. Related structures include graphene antidot lattices, ${ }^{8,9}$ which can be viewed as a periodic network of constrictions. Current state-of-the-art experiments indicate that these may be "sculpted" in monolayer graphene with close to atomic precision to a width of a few benzene rings. ${ }^{10}$

Clearly, for GNCs of this size the current density can locally be very high, and it is important to address their stability and performance under bias. ${ }^{11}$ Experimental results for electron transport, ${ }^{12,13}$ local heating by Raman spectroscopy, ${ }^{14-16}$ and infrared emission, ${ }^{17}$ have been published for GNCs. Recently, it has been argued that several current-induced forces and excitation mechanisms driven by these, besides Joule heating, can play a role in the stability of nanoconductors. ${ }^{18-21}$ In particular, energy nonconservative "wind"/"waterwheel" forces may transfer energy to the phonons in parallel with the well-known Joule heating. However, it is not easy to directly infer these mechanisms from experiments in most cases. On the other hand for graphene, the structural response to a high bias can be studied by in situ transmission electron microscopy, making graphene nanoconductors a good test bed for current-induced phenomena. $^{22-24}$ In particular, a gate electrode can be used to control Fermi level and electronic states involved in the transport, and thereby the current-induced excitation.

In this Rapid Communication, we calculate the currentinduced phonon excitation in a small hydrogen-passivated GNC (Fig. 1) using parameters obtained from density functional theory (DFT). We find a highly nonlinear heating of the GNC due to the deterministic current-induced forces, as opposed to the Joule heating by random forces. In particular, for certain phonon modes in the GNC the nonequilibrium electronic friction force turns into an amplification for voltages beyond a threshold voltage. These amplified modes will dominate the dynamics and lead to a breakdown of the harmonic approximation beyond the voltage threshold. ${ }^{20,21}$ The nonequilibrium negative friction was theoretically predicted for low conductance tunneling transport through asymmetric molecules $^{21,25}$ driven by population inversion between two molecular states. Here the highly conducting GNC displays negative friction at finite bias due to a more generic mechanism, which we trace back to a different coupling to the electronic states involved in the phonon emission and absorption processes.

In Fig. 1(b) we see how the electron transmission of the GNC for energies around the charge neutral Fermi energy $\left(E_{F}=0\right)$ is dominated by two resonance peaks originating from states presenting localized current along the edges (first peak) and through the center (second peak) of the GNC, respectively. Resonances occur due to the diffraction barrier at abrupt interfaces in graphene. ${ }^{13,26}$ The calculated localized voltage drop and high current across the GNC is shown in Figs. 2(a) and 2(b). By employing a gate voltage $\left(V_{g}\right)$ we may tune $E_{F}$ to a highly conducting peak and consider the phonon excitation close to the resonance. We will focus on the constriction gated to the second peak which is mostly unaffected by the boundary conditions in the electrodes $(k$ point sampling), ${ }^{27}$ and exhibits little dependence on the applied bias $\left(V_{a}\right)$ [cf. Fig. 2(c)].

To address the phonon excitation in the presence of current we employ the semiclassical generalized Langevin equation (SCLE). ${ }^{1920,28,29}$ The SCLE describes the Joule heating, current-induced forces, and coupling to electrode phonons in the same formalism. For the mass-scaled ion displacements $(Q)$ the SCLE reads

$$
\ddot{Q}(t)=-K Q(t)-\int^{t} \Pi^{r}\left(t-t^{\prime}\right) Q\left(t^{\prime}\right) d t^{\prime}+f(t)
$$

Here $K$ is the force constant matrix. The couplings to the electron and phonon baths are described by the retarded phonon self-energies $\Pi^{r}=\Pi_{e}^{r}+\Pi_{\mathrm{ph}}^{r}$, and the random noise force, $f(t)$, accounts for the Joule heating. ${ }^{28}$ We consider the retarded self-energy due to the interaction between the 


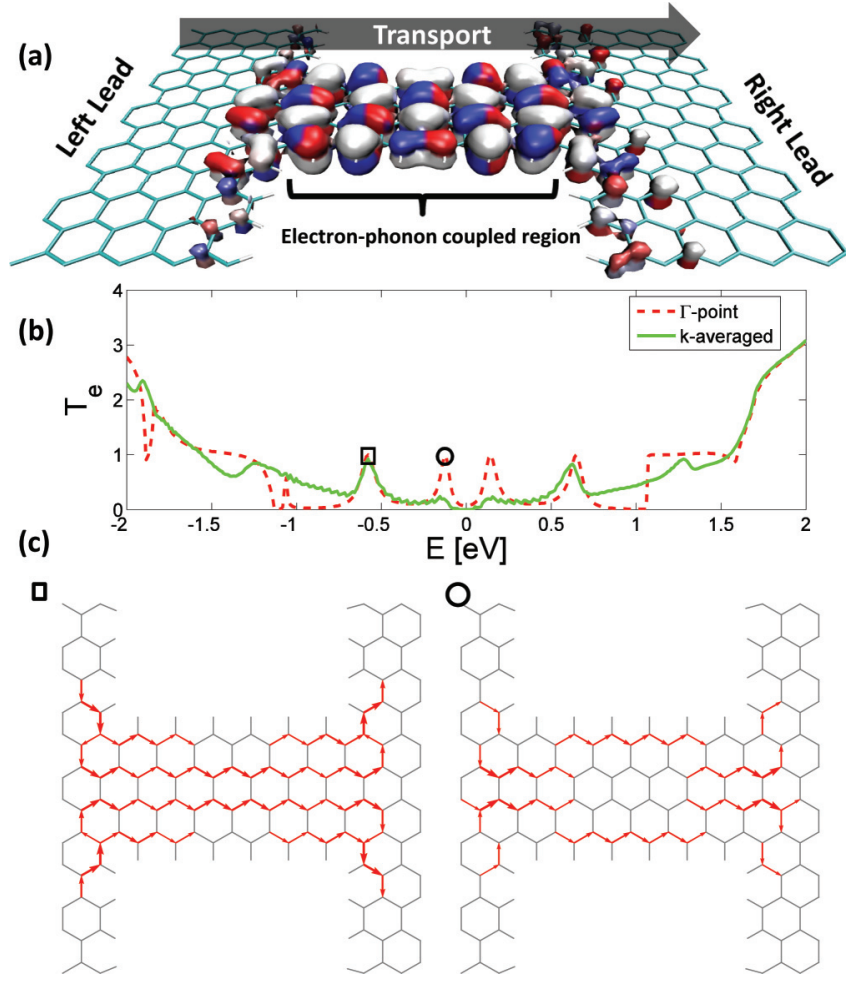

FIG. 1. (Color online) (a) Transport setup illustrating the hydrogen passivated GNC between two semi-infinite graphene leads. The left eigenchannel at zero bias and $E \approx-0.58 \mathrm{eV}$ (colored according to phase, red-white-blue from $-\pi$ to $\pi$ ). (b) $\Gamma$ point and the $k$-averaged transmission function ( $E=0$ corresponds to the Dirac point). (c) Bond currents at the two peaks $(\Gamma)$ marked in the transmission plot $(E \approx-0.12 \mathrm{eV}$ and $E \approx-0.58 \mathrm{eV})$.

phonons and the electronic current,

$$
\begin{aligned}
\Pi_{e}^{r}(\omega)= & i \pi \operatorname{Re} \Lambda(\omega)-\pi \operatorname{Im} \Lambda(\omega) \\
& +\pi \mathcal{H}\left\{\operatorname{Re} \Lambda\left(\omega^{\prime}\right)\right\}(\omega)+i \pi \mathcal{H}\left\{\operatorname{Im} \Lambda\left(\omega^{\prime}\right)\right\}(\omega),
\end{aligned}
$$

(a)

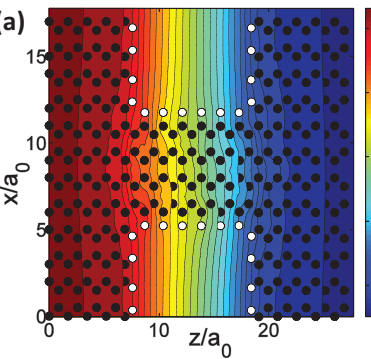

(c)
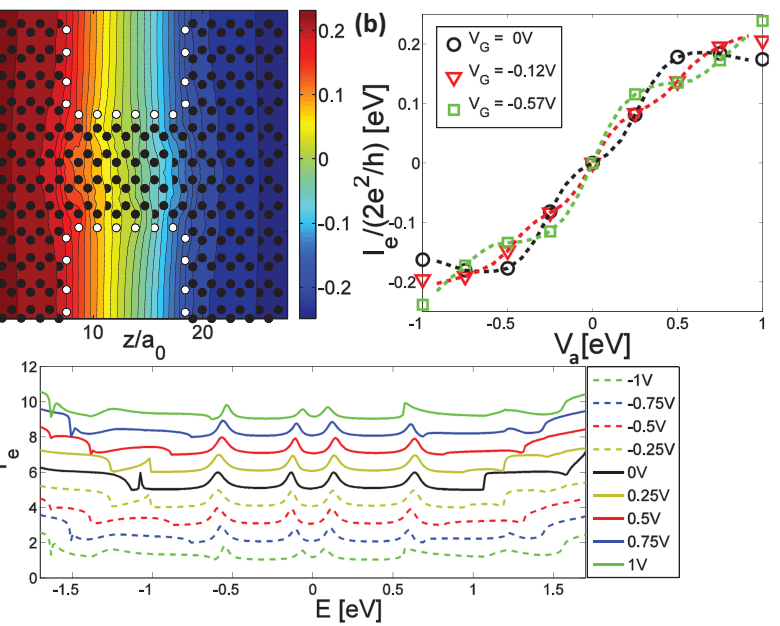

FIG. 2. (Color online) (a) Real space potential drop ( $V_{a}=$ $0.5 \mathrm{eV}$ ) integrated along the out-of-plane direction (in the region with nonvanishing electronic density). (b) $I V$ characteristics for the GNC gated to different chemical potentials. Gating to a peak lowers the resistance at low $V_{a}$. (c) Transmission curves (shifted vertically) for different applied bias $\left(E_{F}=0\right)$. which is given by the interaction-weighted electron-hole pair density of states, $\Lambda$, and its Hilbert transform $(\mathcal{H}){ }^{30}$ The four terms in this expression yield the electronic friction, nonconservative wind, renormalization, and Berry forces in nonequilibrium conditions, respectively. ${ }^{19}$ Especially for the nonequilibrium electron system, $\Lambda=\sum_{\alpha, \beta} \Lambda^{\alpha \beta}$, with contributions from left/right leads $(\alpha=L, R)$,

$$
\begin{aligned}
\Lambda^{\alpha \beta}(\omega) \equiv & 2 \int \frac{d \epsilon}{4 \pi^{2}} \operatorname{Tr}\left[\mathbf{M}^{k} \mathbf{A}_{\alpha}(\epsilon+\omega) \mathbf{M}^{l} \mathbf{A}_{\beta}(\epsilon)\right] \\
& \times\left[n_{F}\left(\epsilon+\omega-\mu_{\alpha}\right)-n_{F}\left(\epsilon-\mu_{\beta}\right)\right] .
\end{aligned}
$$

Here $\mathbf{M}^{k}$ is the coupling to phonon mode $k, \mathbf{A}_{\alpha}$ is the electronic spectral density for states originating from lead $\alpha$ with chemical potential $\mu_{\alpha}$, and $n_{F}$ is the Fermi distribution. The spectral density for the noise, $f$, including the Joule heating, is given by

$$
S_{f}(\omega)=-\pi \sum_{\alpha, \beta} \Lambda^{\alpha \beta}(\omega) \operatorname{coth}\left(\frac{\omega-\left(\mu_{\alpha}-\mu_{\beta}\right)}{2 k_{B} T}\right) .
$$

Importantly, we include the full electronic and phononic structures of the graphene electrodes, and go beyond the constant/wide-band approximation (WBA) for the electronic structure. This is essential for our results of the phonon excitation when the graphene system is gated close to electronic resonance. We determine all parameters entering the SCLE above in the presence of current using first principles DFT and nonequilibrium Green's functions (DFT-NEGF). ${ }^{27,31-33}$ We restrict the el-ph interaction to the GNC region where the current density is high, and evaluate the electronic spectrum at finite bias, but neglect the small voltage dependence of $K$ and $\mathbf{M}^{k}$.

We note that the GNC device region in the present calculation encompasses a basis of 1336 orbitals for the electronic subsystem [matrix size in Eq. (3)]. Thus in order to efficiently compute $\Lambda$ in Eq. (3) beyond WBA we first limited the basis. We employed an expansion of the retarded Green's function and $\mathbf{A}_{\alpha}$ in the eigenspace of $\mathbf{H}+\Sigma_{0}\left(E_{F}\right), \mathbf{H}$ being the electronic Hamiltonian and $\Sigma_{0}\left(E_{F}\right)$ the lead self-energies, which vary slowly with energy. ${ }^{34}$ We have found it sufficient to limit this basis to 200 states within the interval $[-7,6] \mathrm{eV}$ around $E_{F}$. Secondly, we computed $\Lambda$ by parallel execution over the $\omega$ and $V_{a}$ parameters. The phonon self-energies describing the semi-infinite graphene leads was calculated on the basis on a zero-bias finite difference calculation. Detailed information is given in the Supplemental Material. ${ }^{35}$

From Eq. (1) we can obtain the nonequilibrium retarded phonon Green's function,

$$
D^{r}(\omega)=\left[D^{a}(\omega)\right]^{\dagger}=\left[(\omega+i \eta)^{2}-K-\Pi^{r}(\omega)\right]^{-1},
$$

and the excitation in terms of the average kinetic energy of the phonons,

$$
E_{\mathrm{kin}}=\int_{-\infty}^{\infty} \frac{d \omega}{2 \pi} \omega^{2} \operatorname{Tr}\left[D^{r}(\omega) S_{f}(\omega) D^{a}(\omega)\right] .
$$

The phonon density of states (DOS) is given by $-2 / \pi \omega \operatorname{Im}\left[D^{r}(\omega)\right]$. The DOS is affected both by the coupling to electrons, in particular giving rise to nonequilibrium forces, as well as coupling to the electrode phonons. In Fig. 3(a) we show the phonon DOS at applied bias of $V_{a}=0$ and 

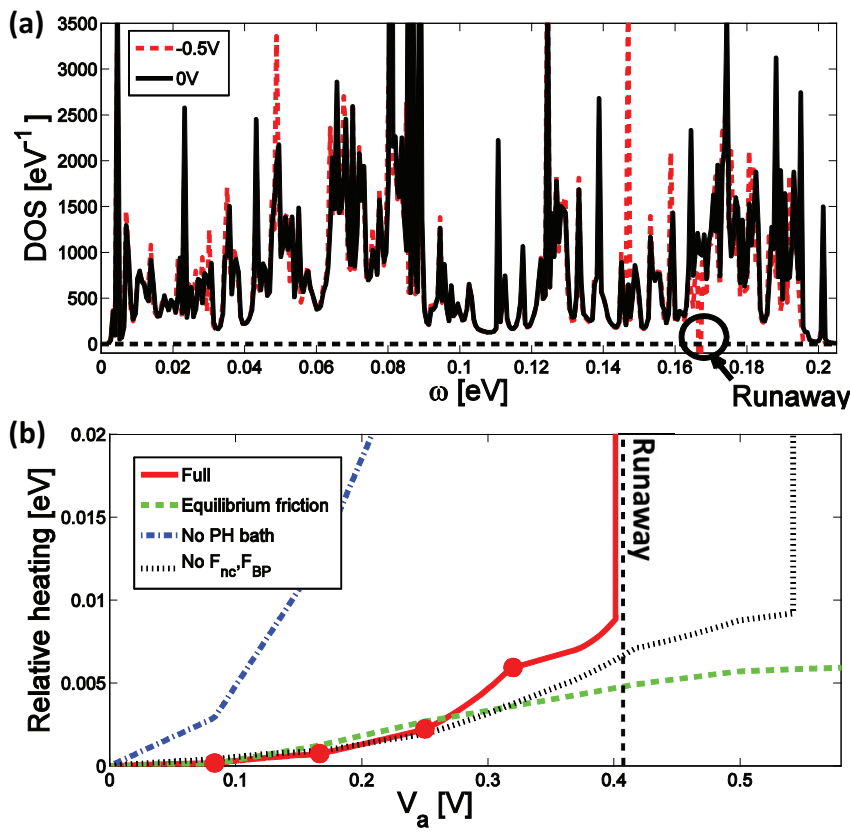

FIG. 3. (Color online) (a) Dashed (full) lines show the phonon DOS of the GNC with (without) electronic current. An unstable "runaway" mode appears for an applied bias of $V_{a} \approx \pm 0.5 \mathrm{~V}$ as a negative DOS peak. (b) Heating (change in average kinetic energy per atom due to current) of the GNC at $300 \mathrm{~K}$. Full line: Result including all current-induced forces. Dashed line: only fluctuating force (Joule heating) and zero-bias electronic friction. Dot-dashed line: The wide-band approximation without coupling to the electrode phonon bath. Dotted line: Full calculation neglecting the nonconservative wind and Berry-phase forces.

$V_{a}=0.5 \mathrm{~V}$. Most importantly, the DOS becomes negative at a particular phonon frequency $(\omega \approx 170 \mathrm{meV})$, corresponding to a negatively damped mode, denoted "runaway". From Eq. (5) the runaway gives rise to a divergence in the currentinduced change of $E_{\text {kin }}$ (heating) of the GNC at $V_{a} \approx 0.4 \mathrm{~V}$ [see Fig. 3(b)]. This signifies an instability in the harmonic approximation, where the high excitation is likely to lead to dramatic effects such as contact disruption. ${ }^{18}$

The instability can be traced back to the bias dependent electronic friction, and disappears when this is kept at its zero-bias value. We further note that for $V_{a}$ above $\sim 0.3 \mathrm{~V}$ the deterministic current-induced forces lead to a qualitatively different heating compared to that of Joule heating only. Figure 3(b) furthermore shows how the damping due to electrode phonons is crucial: The heating increases by an order of magnitude if the electrode-phonon bath is neglected. Moreover, if we neglect the damping due to the phonon bath we observe runaway starting already at $V_{a} \approx 0.15 \mathrm{~V}$, increasing to more than 15 runaway modes at $V_{a} \approx 0.4 \mathrm{~V}$, both due to the effects of negative friction and nonconservative forces. ${ }^{18}$ The nonconservative wind and Berry-phase forces are found to be on the same order of magnitude for the runaway mode. Even though they do not themselves lead to the first runaway condition they lower the runaway threshold.

We will now in detail analyze the origin of the runaway. We focus on the modes contributing to the phonon DOS peak around the runaway, $\omega_{0} \approx 170 \mathrm{meV}$. They can be found as

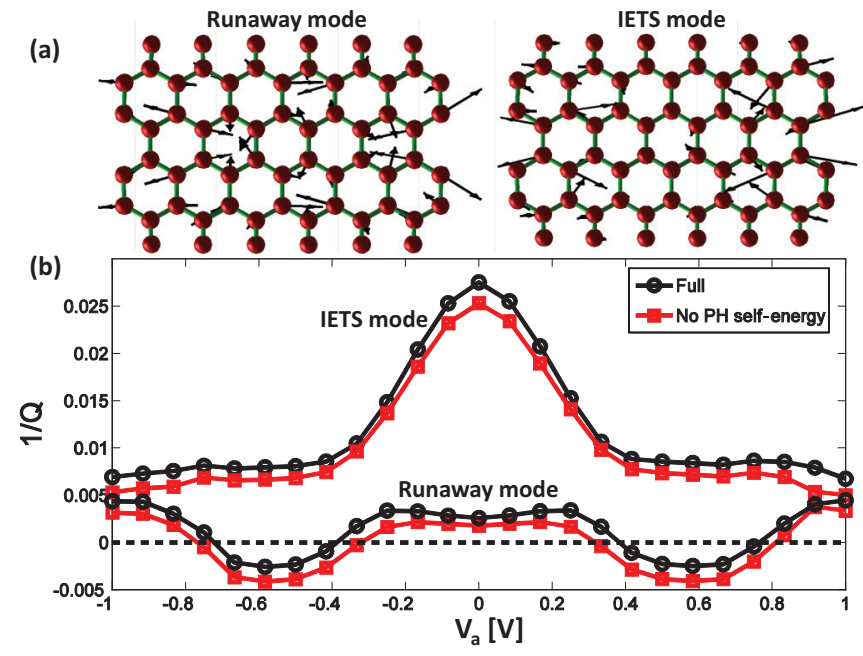

FIG. 4. (Color online) (a) Two degenerate modes ("runaway"/ "IETS") at $V_{a}=0.4 \mathrm{~V}$ with $\omega_{0} \approx 170 \mathrm{meV}$. The runaway mode breaks the left-right symmetry due to the coupling to the nonequilibrium electrons and becomes unstable at finite bias. The IETS yields the largest inelastic signal in the current. (b) Inverse $Q$ factor (loss) as a function of bias for the modes.

the eigenvectors of $K+\operatorname{Re} \Pi^{r}\left(\omega_{0}\right)$. The two main modes are displayed in Fig. 4. The "IETS mode" exhibits the largest inelastic tunnel spectroscopy signal (IETS) in the electronic current and largest noise $S_{f, i i}\left(\omega_{i}\right)$, while the "runaway mode" is the first mode that turns unstable with increasing $V_{a}$. In Fig. 4 we show the inverse quality factor $1 / Q=-2 \frac{\operatorname{Im}(\omega)}{\operatorname{Re}(\omega)}=$ $1 / Q_{\mathrm{ph}}+1 / Q_{\mathrm{el}}\left(V_{a}\right)$ (energy loss/period) for the two modes as a function of $V_{a}$. The $Q_{\mathrm{ph}}$ factor is relatively big, especially for the runaway mode, due to low phonon DOS around $\omega_{0}$. The runaway corresponds to amplification $1 / Q<0$, while $1 / Q>0$ remains for the IETS mode despite a strong decrease with bias.

It is instructive to view the runaway in terms of phonon absorption/emission processes in a simple master equation for the phonon number $N$,

$$
\dot{N}=\mathcal{B}(N+1)-\mathcal{A} N,
$$

where $\mathcal{A}(\mathcal{B})$ are the rates for absorption (emission). From Fermi's golden rule we find the emission,

$$
\mathcal{B}=-2 \pi \sum_{\alpha \beta} n_{B}\left(\hbar \omega_{0}+\mu_{\alpha}-\mu_{\beta}\right) \Lambda^{\beta \alpha}\left(\omega_{0}\right),
$$

and $\mathcal{A}$ is obtained by a replacement $\omega \rightarrow-\omega$. Only a single scattering state $\left|\psi_{L / R}\right\rangle$ contributes to $\mathcal{A}$ and $\mathcal{B}$. Expressed in the single flux-normalized eigenchannel, and assuming $k_{B} T \ll$ $\hbar \omega_{0}<e V_{a}$, we have

$$
\begin{aligned}
& \mathcal{B} \approx \int_{\mu_{R}+\hbar \omega_{0}}^{\mu_{L}}\left|\left\langle\psi_{L}(\epsilon)|\mathbf{M}| \psi_{R}\left(\epsilon-\hbar \omega_{0}\right)\right\rangle\right|^{2} \frac{d \epsilon}{2 \pi}, \\
& \mathcal{A} \approx \int_{\mu_{R}-\hbar \omega_{0}}^{\mu_{L}}\left|\left\langle\psi_{L}(\epsilon)|\mathbf{M}| \psi_{R}\left(\epsilon+\hbar \omega_{0}\right)\right\rangle\right|^{2} \frac{d \epsilon}{2 \pi} .
\end{aligned}
$$

Here we did not include the intraelectrode terms $\left(\Lambda^{L L / R R}\right)$ in $\mathcal{A}$ since these vary only slightly with $V_{a}$ for the runaway mode. The phonon absorption rate decreases while the emission rate increases as the bias exceeds the mode frequency [see 

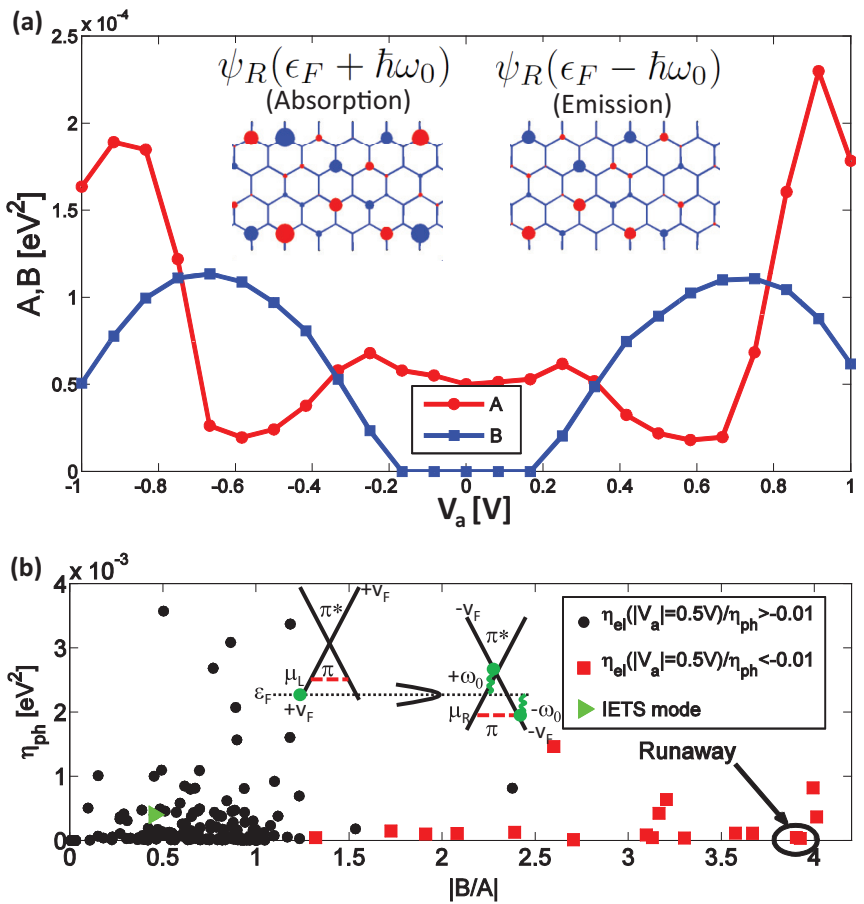

FIG. 5. (Color online) Nonequilibrium friction mechanism. (a) Phonon absorption/emission $(\mathcal{A} / \mathcal{B})$ rates for the runaway mode. Note that $\mathcal{B}=0$ for $V_{a}<\omega_{0}$. When $V_{a}> \pm 0.4 \mathrm{~V}$ emission exceeds absorption, $\mathcal{B}>\mathcal{A}$. Inset: at resonance scattering states giving the main contribution to the interaction integrals. The radius shows the absolute value $|\psi(x, y)|$ of the eigenstate, while the color indicates the sign of the real part. (b) Runaway occurs for the mode with the largest emission and lowest phonon friction $\left(\eta_{\mathrm{ph}}\right)$. Squares indicate modes with a significant electron friction $\left(\eta_{\mathrm{el}}\right)$. These modes all have $\mathcal{A}, \mathcal{B}$ coefficients with the same behavior as the first runaway mode. Inset: resonance between two graphene leads at certain filling (red dashed line) and bias voltage. An incoming scattering state (left green dot) at resonance (dashed line) can either absorb $\left(+\omega_{0}\right)$ to a state with lower DOS close to the $\pi-\pi^{*}$ crossing or change to a state with higher DOS by emission $\left(-\omega_{0}\right)$.

Fig. 5(a)]. The electronic friction is given by the difference $\mathcal{A}-\mathcal{B}=-2 \pi \sum_{\alpha \beta} \Lambda^{\alpha \beta}(\omega)$. This difference manifests itself in how the $Q$ factor varies with bias for the runaway mode. The state symmetry changes significantly with energy [Fig. 1(c)]. Thus we expect that a given phonon will yield very different emission and absorption matrix elements due to the symmetry. The symmetry of the scattering state $\psi_{L}^{*}\left(E_{F}\right)$ is almost unchanged from going up in energy (absorption) [see $\psi_{R}\left(E_{F}+\omega_{0}\right)$ in the inset of Fig. 5(a)], while the symmetry of $\psi_{R}\left(E_{F}-\omega_{0}\right)$ differs significantly from this. In particular, the el-ph matrix element of the runaway mode yields very low absorption and high emission due to the selective symmetry of this phonon mode. The large phonon frequencies and linear DOS of graphene strengthens this symmetry breaking. The negative electronic friction is found for several modes and seems to be a generic phenomena in graphene nanostructures.

In Fig. 5(b) we illustrate how each mode shows up in a parameter space of the phonon friction and $\mathcal{B} / \mathcal{A}$. The dominating runaway mode shows up at high $\mathcal{B} / \mathcal{A}$ and low phonon friction. The other modes with a nonvanishing negative electron friction are also displayed. All these modes have $\mathcal{A}, \mathcal{B}$ coefficients with the same generic behavior as the first runaway mode [Fig. 5(a)]. In the general case where one has a resonance between graphene leads [inset of Fig. 5(b)], the wave incoming at resonance will absorb to an eigenstate close to the Dirac crossing. Hence it will have low DOS and a dissimilar phase. On the contrary emission leads to an eigenstate with larger DOS and similar phase. This holds true for states dominated by the interlead contributions. Compared to the runaway mode the IETS mode has low emission-absorption ratio due to high intraelectrode terms $\Lambda^{L L / R R}$ and a higher phonon damping.

We conclude that negative friction can appear for certain phonons in realistic systems such as graphene nanoconstrictions in the presence of electrical current. The negative friction effect is here rooted in the high phonon energies which lead to markedly different symmetry of the electronic states involved in emission and absorption and thus different matrix elements and rates. Therefore, it is a generic nonequilibrium effect. Two-dimensional systems like graphene, where a gate can be applied, make an exciting test bed for probing effects of electronic current on the atomic scale.

We thank the Danish Center for Scientific Computing (DCSC) for providing computer resources. The Center for Nanostructured Graphene (CNG) is sponsored by the Danish National Research Foundation. *tue.gunst@ nanotech.dtu.dk

${ }^{\dagger}$ Present address: School of Physics, Huazhong University of Science and Technology, Wuhan, China; jtlu@ mail.hust.edu.cn

${ }^{1}$ K. S. Novoselov, A. K. Geim, S. V. Morozov, D. Jiang, Y. Zhang, S. V. Dubonos, I. V. Grigorieva, and A. A. Firsov, Science 306, 666 (2004).

${ }^{2}$ A. H. Castro Neto, F. Guinea, N. M. R. Peres, K. S. Novoselov, and A. K. Geim, Rev. Mod. Phys. 81, 109 (2009).

${ }^{3}$ A. K. Geim, Science 324, 1530 (2009).

${ }^{4}$ K. Nakada, M. Fujita, G. Dresselhaus, and M. S. Dresselhaus, Phys. Rev. B 54, 17954 (1996).

${ }^{5}$ L. Brey and H. A. Fertig, Phys. Rev. B 73, 235411 (2006).

${ }^{6}$ D. A. Areshkin and C. T. White, Nano Lett. 7, 3253 (2007).
${ }^{7}$ A. R. Botello-Mendez, E. Cruz-Silva, J. M. Romo-Herrera, F. Lopez-Urias, M. Terrones, B. G. Sumpter, H. Terrones, J.-C. Charlier, and V. Meunier, Nano Lett. 11, 3058 (2011).

${ }^{8}$ J. Bai, X. Zhong, S. Jiang, Y. Huang, and X. Duan, Nat. Nanotech. 5, 190 (2010).

${ }^{9}$ T. G. Pedersen, C. Flindt, J. Pedersen, N. A. Mortensen, A. P. Jauho, and K. Pedersen, Phys. Rev. Lett. 100, 136804 (2008).

${ }^{10}$ Q. Xu, M.-Y. Wu, G. F. Schneider, L. Houben, S. K. Malladi, C. Dekker, E. Yucelen, R. E. Dunin-Borkowski, and H. W. Zandbergen, ACS Nano 7, 1566 (2013).

${ }^{11}$ F. Börrnert, A. Barreiro, D. Wolf, M. I. Katsnelson, B. Büchner, L. M. K. Vandersypen, and M. H. Rümmeli, Nano Lett. 12, 4455 (2012). 
${ }^{12}$ N. Tombros, A. Veligura, J. Junesch, M. H. D. Guimares, I. J. Vera-Marun, H. T. Jonkman, and B. J. v. Wees, Nat. Phys. 7, 697 (2011).

${ }^{13}$ P. Darancet, V. Olevano, and D. Mayou, Phys. Rev. Lett. 102, 136803 (2009).

${ }^{14}$ D.-H. Chae, B. Krauss, K. von Klitzing, and J. H. Smet, Nano Lett. 10, 466 (2009).

${ }^{15}$ S. Berciaud, M. Y. Han, K. F. Mak, L. E. Brus, P. Kim, and T. F. Heinz, Phys. Rev. Lett. 104, 227401 (2010).

${ }^{16}$ I. Jo, I.-K. Hsu, Y. J. Lee, M. M. Sadeghi, S. Kim, S. Cronin, E. Tutuc, S. K. Banerjee, Z. Yao, and L. Shi, Nano Lett. 11, 85 (2010).

${ }^{17}$ M. Freitag, H.-Y. Chiu, M. Steiner, V. Perebeinos, and P. Avouris, Nat. Nanotech. 5, 497 (2010).

${ }^{18}$ D. Dundas, E. J. McEniry, and T. N. Todorov, Nat. Nanotech. 4, 99 (2009).

${ }^{19}$ J.-T. Lü, M. Brandbyge, P. Hedegård, T. N. Todorov, and D. Dundas, Phys. Rev. B 85, 245444 (2012).

${ }^{20}$ N. Bode, S. V. Kusminskiy, R. Egger, and F. von Oppen, Phys. Rev. Lett. 107, 036804 (2011).

${ }^{21}$ J.-T. Lü, P. Hedegård, and M. Brandbyge, Phys. Rev. Lett. 107, 046801 (2011).

${ }^{22}$ X. Jia, M. Hofmann, V. Meunier, B. G. Sumpter, J. CamposDelgado, J. M. Romo-Herrera, H. Son, Y.-P. Hsieh, A. Reina, J. Kong, M. Terrones, and M. S. Dresselhaus, Science 323, 1701 (2009).

${ }^{23}$ A. Barreiro, F. Börrnert, M. H. Rümmeli, B. Büchner, and L. M. K. Vandersypen, Nano Lett. 12, 1873 (2012).

${ }^{24}$ M. Engelund, J. A. Fürst, A. P. Jauho, and M. Brandbyge, Phys. Rev. Lett. 104, 036807 (2010).
${ }^{25}$ D. A. Ryndyk, M. Hartung, and G. Cuniberti, Phys. Rev. B 73, 045420 (2006).

${ }^{26}$ S. Ihnatsenka and G. Kirczenow, Phys. Rev. B 85, 121407 (2012).

${ }^{27}$ M. Brandbyge, J.-L. Mozos, P. Ordejon, J. Taylor, and K. Stokbro, Phys. Rev. B 65, 165401 (2002).

${ }^{28}$ J.-T. Lü, M. Brandbyge, and P. Hedegård, Nano Lett. 10, 1657 (2010).

${ }^{29}$ J.-T. Lü, T. Gunst, P. Hedegård, and M. Brandbyge, Beilstein J. Nanotechnol. 2, 814 (2011).

${ }^{30}$ The Hilbert transform is defined as $\mathcal{H}\left\{g\left(x^{\prime}\right)\right\}(x)=\frac{1}{\pi} \mathcal{P} \int \frac{g\left(x^{\prime}\right)}{x^{\prime}-x} d x^{\prime}$.

${ }^{31}$ J. M. Soler, E. Artacho, J. D. Gale, A. Garca, J. Junquera, P. Ordejn, and D. Snchez-Portal, J. Phys.: Condens. Matter 14, 2745 (2002).

${ }^{32}$ T. Frederiksen, M. Paulsson, M. Brandbyge, and A.-P. Jauho, Phys. Rev. B 75, 205413 (2007).

${ }^{33}$ We used single-zeta-polarized localized pseudoatomic orbitals in the basis set for both hydrogen and carbon. Exchange-correlation effects were handled within generalized gradient approximation Perdew-Burke-Ernzerhof and a grid cutoff of 210 Ry was applied. The Brillouin zone was sampled with a [5 11 2] Monkhorst-Pack grid, with the $x$ axis as the transverse direction and the $z$ axis as the transport direction. The system was relaxed until all forces were smaller than $0.005 \mathrm{eV} /$ Ang. We used 150 points along the bias contour with a broadening of $0.01 \mathrm{eV}$.

${ }^{34}$ R. Egger and A. O. Gogolin, Phys. Rev. B 77, 113405 (2008).

${ }^{35}$ See Supplemental Material at http://link.aps.org/supplemental/ 10.1103/PhysRevB.88.161401 for detailed information on the electron and phonon DFT-NEGF modelling. 